\title{
Stochastic Programming for Optimizing Bidding Strategies of a Nordic Hydropower Producer
}

\author{
${ }^{a}$ Stein-Erik Fleten, ${ }^{b}$ Trine Krogh Kristoffersen* \\ ${ }^{a}$ Department of Industrial Economics and Technology Management, \\ Norwegian University of Science and Technology, NO-7491 Trondheim, \\ stein-erik.fleten@iot.ntnu.no \\ ${ }^{b}$ Department of Operations Research, University of Aarhus, \\ DK-8000 Aarhus C, trinek@imf.au.dk
}

Working paper, September 22 2005. This version April 32006.

\begin{abstract}
From the point of view of a price-taking hydropower producer participating in the dayahead power market, market prices are highly uncertain. The present paper provides a model for determining optimal bidding strategies taking into account this uncertainty. In particular, realistic market price scenarios are generated and a stochastic mixed-integer linear programming model that takes in both production and physical trading aspects is developed. The idea is to explore the effects of including uncertainty into the optimization model and to compare the stochastic approach to a deterministic one. The model is illustrated with data from a Norwegian hydropower producer and the Nordic power market at Nord Pool.
\end{abstract}

Keywords: OR in energy, electricity markets; bidding; stochastic programming; scenarios

\section{Introduction}

The increased interest in power optimization problems within recent years has been stimulated by the tendency to decentralize and deregulate the power sector. Whereas traditional planning and operating procedures were based on centralized optimization, novel ones rest on independent optimization of separate power plants. As an attempt to increase efficiency, markets have been liberalized forcing former procedures to conform to more market oriented ones.

With the pioneering act of 1990 Norway was one of the first countries in the world to deregulate and already in 1991 a Norwegian power market was established. From 1996 to 2000 the national Norwegian power market developed into a multi-national Nordic power market encompassing also the three neighboring countries, Sweden, Finland and Denmark. Today, the Nordic market has successfully adapted to the new competitive environment and serves as a model for restructuring other power markets. An important component of the power market is the presence of the

${ }^{*}$ Corresponding author. Email: trinek@imf.au.dk 
power exchange facilitating physical trading and being immediately effectively. The spot market, Elspot, at Nord Pool, takes the form of a pool-based market in which market participants exchange power contracts for physical delivery the following operation day and is referred to as the day-ahead market. In 2004 a total of 167 TWh was exchanged at Elspot, representing $42 \%$ ${ }^{1}$ percent of overall consumption in the Nordic region.

Inevitably, the physical trading becomes a means of growing importance in the economic activity of the power sector with the so-called bidding problem being a major challenge in this respect. Indeed, bidding involves submitting sales and purchase bids to the power exchange one day in advance. However, in clearing the market, price calculations are based on the balance between total sales and purchases, making prices unknown at the time of bidding. If price uncertainty is high, intelligent bidding is rather complicated. The need for an approach to determine optimal bidding strategies is obvious.

In the Nordic area, power production is diverse comprising nuclear, thermal as well as hydro and other kinds of renewable power such as wind. Sweden and Finland use nuclear, fossil-fuel and hydropower production, Denmark mainly produces from conventional thermal power plants as well as combined heat and power facilities and accounts for most of the wind power, while generation in Norway is close to being hydropower exclusively. The number of hydropower producers participating in Nord Pool amounts to around 48, the total hydro-power based generation in the Nordic area being 183 TWh or $54 \%$ of the total generation in $2004^{2}$. In general, thermal power production is located in the south, whereas hydropower production is found in the northern parts of the Nordic area and is transmitted to the heavily populated south. Approving the comparatively low costs of hydropower production, the market prefers this energy source over thermal power.

A crucial difference between thermal power and hydropower production is the possibility of storing energy. If disposing of production in the power market, storing makes it feasible to produce when price conditions are propitious. That is, when prices are high, the energy is sold immediately, when prices are low, however, the energy is saved for later disposal at higher prices. This degree of flexibility makes bidding strategies extremely relevant for hydropower producers.

As already indicated, bidding and production has to be coordinated and cannot be considered separately. Thus, considering the bidding problem, both pool exchange and production aspects should be taken into account. This makes the decision process of the bidding problem divisible into stages. Indeed, bidding decisions take place with probabilistic information on market prices, whereas production decisions are deferrable making it possible to react to new information. Hence, the problem fits the framework of two-stage stochastic programming. More specifically, the bidding problem is formulated as a two-stage stochastic mixed-integer linear program. The objective is to maximize sales and production profits subject to a number of bidding and hydrological constraints. First-stage concerns the day-ahead bidding, whereas second-stage takes in the real-time production. Uncertainty is represented by a known probability distribution which is obtained modeling the stochastic process of prices as a nonparametric or parametric stochastic model calibrated from historical data or based on past experience and future expectations. In practice such stochastic models are often available for forecasting. For other power optimization

\footnotetext{
${ }^{1}$ Reference: www.nordel.org and www.nordpool.no

${ }^{2}$ Reference: www.nordel.org
} 
models in stochastic programming, see [28].

In practice short-term power planning and operation are often based on deterministic optimization tools, such as the Short-term Hydro Operation Planning (SHOP) model [8] used in Norway, whereas setting up bidding tables has rested on skills and experience of the operating engineers. Until now, taking price uncertainty into consideration has not been done explicitly neither in planning production nor when bidding. In the process of bidding, however, price uncertainty is indeed acknowledged and managed to some extent through the use of bidding curves that assign different volumes to different prices.

One of the aims of the present paper is to compare the stochastic approach with a deterministic one. In particular, we investigate how the inclusion of uncertainty into the optimization model affects the objective function value, i.e. the sales and production profit, as well as the solution, i.e. the structure of the bids.

It is anticipated that expected profits are higher when taking a stochastic approach rather than a deterministic one. Moreover, it is expected that the structure of the bids in a stochastic solution will differ from the structure of the bids in a deterministic one. A deterministic model will suggest to use hourly bids only, whereas a stochastic model will propose to combine hourly bids and block bids. Indeed, if the day-ahead market prices are known in advance, hourly bids dominate block bids as these bids can adjust hourly to price variations. On the other hand, if the day-ahead market prices are not known at the time of bidding, block bids can be used to protect against unexpected price changes over time.

Although the paper is motivated by needs of small Nordic hydropower producers the bidding model should be applicable to other price-taking power producers acting in a pool-based dayahead market accepting piecewise linear bids and block bids.

Considering the literature, other contributions that deal with the bidding problem include the following. The paper [5] addresses the problem from the perspective of a price-taking thermal power producer bidding in the day-ahead market under market price uncertainty. Based on time series analysis and forecasts of market prices, a simple bidding strategy is developed. Essentially, the expected value problem is solved, i.e. the stochastic prices are replaced by expected prices, and the optimal solution is used to construct piecewise constant bidding curves. For comparison, our model does not aggregate information but utilizes all information on uncertainty. In a spirit similar to the present paper, [9] proposes a stochastic programming formulation of the bidding problem for a price-taking power marketer who supplies power to end users through purchases in the day-ahead market. Solving the problem, the result is piecewise linear bidding curves. Again, bidding is subject to market price uncertainty. Uncertainty is represented by scenarios that, in contrast to our paper, are generated using moment matching. Another stochastic programming approach related to the bidding problem appears in [22]. There, a model for the simultaneous planning of production and day-ahead energy sales of a hydropower plant is given and hence, the output includes both production and bidding schedules. As opposed to the model here, the plant is not a price-taker and the structure of the bidding process is incorporated directly in the model so that uncertainty concerns the competitor's bids. Likewise, [29] and [20] build linear bidding curves for competitive suppliers in a day-ahead power market who do not have full information on the competitor's bidding curves. The latter is handled using estimates of the bidding curves, such estimates being based on expectations and correlations. The authors suggest using 
maximum profit bidding strategies and, if not feasible for dispatch, minimum output bidding strategies. [1] studies strategies of generators making offers into power markets in circumstances where uncertainty also concerns the behavior of other market participants in a competitive environment. Uncertainty is modeled by a so-called market distribution and it is shown how to derive smooth bidding curves solving an optimal control problem. It should be stressed that unlike all previous studies, our model allows for both piecewise linear bids and block bids.

As regards hydro power problems in the literature, we give a few references. [7] forms an optimal control problem that relates to the short-term operation of a hydropower plant with reservoirs in a cascade. Inflows are unknown. Assuming a known probability distribution, the problem can be reformulated as a multi-stage stochastic program. [23] develops a deterministic short-term optimization model for a power system comprising both thermal and hydro production, whereas [22] presents a stochastic version of the like. Finally, [24] considers hydropower unit commitment subject to uncertain demand in a multi-stage stochastic programming framework.

The outline of the paper is as follows. We explain the composition of the day-ahead market and model the bidding process in Section 1. In Section 2 we model the hydropower production plant in a relatively standard way. Section 3 is devoted to scenario generation, whereas Section 4 illustrates with a case study from a Norwegian hydropower producer and the Nordic power exchange, Nord Pool.

\section{Day-ahead bidding}

Elspot at Nord Pool is a spot market in which contracts for physical delivery the following operation day are exchanged. The power exchange offers access to the physical market at low transaction costs as well as a possibility of settlement close to real-time operation. The Elspot contracts are power obligations to deliver or receive power of a duration of one hour or longer. To be specific, contracts are divided into hourly bids, block bids and flexible hourly bids. All bids consist of a price and a volume. Submitting hourly bids, sales bids have to be listed in ascending order and purchase bids in descending order according to price. Consistent with the rules, for each hour Elspot will make a linear interpolation between the price-volume points to construct the bidding curve. The volume dispatched is determined by the point on the bidding curve that corresponds to the market price. All transactions are settled at the market price. Block bids are aggregated bids valid for a number of consecutive hours and associated with only one price and volume. What is referred to as the mean price condition determines whether a block bid is either rejected or accepted as a whole. If the price of a sales bid is below the average market price or if the price of a purchase bid is above the average market price of the hours specified, the bid is accepted. All transactions are settled at the mean price. Flexible hourly bids are hourly bids that are accepted in the hour with the highest price if this price exceeds a threshold price of the bid. Such bids are omitted here as the bids are mainly used by companies able to close down power intensive production for the hour in question. Participants post the price-differentiated bids for all hours of the following operation day before deadline at noon.

It is important to note that the volumes dispatched and the prices at which transactions are settled are unknown until the market has been cleared and market clearing prices have been determined. Once this is done, each participant receives a notification of the plan. The market 
price calculations are the same for each individual hour. When determining the market price, the bidding curves, these being either sales or purchase curves, are collocated to an aggregated demand curve and an aggregated supply curve. The intersection of the demand curve and the supply curve defines a candidate of the unconstrained market price. Through an iterative process, the bidding curves are updated according to certain priority rules by including those block bids and flexible hourly bids that are accepted and a new unconstrained market price is found. The Nordic region is divided into fixed price zones, Sweden, Finland, East and West Denmark each being one zone and Norway divided into four zones. If the contractual flow between zones does not exceed the grid capacity allocated, the unconstrained market price simply applies to all zones. Otherwise, separate prices are established through counter purchases and corresponding iterations of price calculations in order to relieve grid congestion. In this application we neglect grid congestion, which is the case if for instance all power exchange is local. Hence, there is only one market price.

The following model and strategies should work as a tool of a price-taking hydropower producer for planning how to commit in the power market and how to produce in accordance. It is assumed that the hydropower producer does not participate in bilateral exchange as this do not affect the problem of bidding, but disposes of the entire production in the day-ahead market and the real-time market.

The time horizon of 24 hours is divided into hourly time intervals and denoted $\mathcal{T}=\{1, \ldots, T\}$. From this the set of blocks $\mathcal{B}=\left\{b_{1}, \ldots, b_{B}\right\}$ is constructed, a block being a number minimum two of consecutive hours and the total number of such blocks within 24 hours being $B=276$. Examples of blocks are $b_{140}=\{1, \ldots, 7\}, b_{165}=\{8, \ldots, 18\}$ and $b_{265}=\{19, \ldots, 24\}$.

Regarding the modeling of the bidding process, the problem of selecting both bid prices and bid volumes is nonlinear. However, it has been chosen to work with a linear model, the reason being that the problem formulated as a mixed-integer linear program is rather hard to solve in terms of computing times, that standard software packages are available for solving the problem and that the problem is amenable to special designed stochastic programming algorithms. Hence, for computational reasons, nonlinearities are avoided by fixing prices in advance so that only volumes have to be selected. Let the indices $\mathcal{I}=\{1, \ldots, I\}$ signify the possible prices and denote these prices $p_{i}, i \in \mathcal{I}$ where $p_{i} \leq p_{i+1}$. The corresponding bid volumes are represented by the variables $x_{i t} \in \mathbb{R}_{+}, i \in \mathcal{I}, t \in \mathcal{I}$ for hourly bids and $x_{i b} \in \mathbb{R}_{+}, i \in \mathcal{I}, b \in \mathcal{B}$ for block bids. The variables $y_{t} \in \mathbb{R}_{+}, t \in \mathcal{T}$ and $y_{b} \in \mathbb{R}_{+}, b \in \mathcal{B}$ are the volumes dispatched for hourly bids and block bids. The hourly market prices are denoted $\rho_{t}, t \in \mathcal{T}$ and average market prices for the blocks $\bar{\rho}_{b}, b \in \mathcal{B}$, where $\bar{\rho}_{b}=(1 /|b|) \sum_{t \in b} \rho_{t}$.

Disposing of the hydropower production in the day-ahead market, total sales revenues accumulate to

$$
\sum_{t \in \mathcal{T}} \rho_{t} y_{t}+\sum_{b \in \mathcal{B}} \bar{\rho}_{b} y_{b}
$$

To deal with hourly bids, proceed as in [9]. For each hour $t \in \mathcal{T}$, the bids $\left(x_{i t}, p_{i}\right), i \in \mathcal{I}$ are interpreted as price-volume points on a bidding curve. Constructing the curve, a linear interpolation between the points is made, the result being a piecewise linear curve. Thus, in 
terms of prices, the bidding curve can be expressed as

$$
\rho_{t}= \begin{cases}p_{1}+\frac{p_{2}-p_{1}}{x_{2 t}-x_{1 t}}\left(y_{t}-x_{1 t}\right) & \text {, if } x_{1 t} \leq y_{t}<x_{2 t} \\ \vdots & \\ p_{i-1}+\frac{p_{i}-p_{i-1}}{x_{i t}-x_{i-1 t}}\left(y_{t}-x_{i-1 t}\right) & \text {,if } x_{i-1 t} \leq y_{t}<x_{i t} \\ \vdots & \\ p_{I-1}+\frac{p_{I t}-p_{I-1 t}}{x_{I t}-x_{I-1 t}}\left(y_{t}-x_{I-1 t}\right) & , \text { if } x_{I-1 t} \leq y_{t} \leq x_{I t}\end{cases}
$$

or equivalently, in terms of volumes,

$$
y_{t}= \begin{cases}\frac{\rho_{t}-p_{1}}{p_{2}-p_{1}} x_{2 t}+\frac{p_{2}-\rho_{t}}{p_{2}-p_{1}} x_{1 t} & \text {,if } p_{1} \leq \rho_{t}<p_{2} \\ \vdots & \\ \frac{\rho_{t}-p_{i-1}}{p_{i}-p_{i-1}} x_{i t}+\frac{p_{i}-\rho_{t}}{p_{i}-p_{i-1}} x_{i-1 t} & \text {,if } p_{i-1} \leq \rho_{t}<p_{i} \\ \vdots & \\ \frac{\rho_{t}-p_{I-1}}{p_{I}-p_{I-1}} x_{I-1 t}+\frac{p_{I}-\rho_{t}}{p_{I}-p_{I-1}} x_{I t} & \text {,if } p_{I-1} \leq \rho_{t} \leq p_{I} .\end{cases}
$$

Considering sales bids, it would be natural for the bidding curve to be increasing, i.e. ${ }^{3}$

$$
x_{i t} \leq x_{i+1 t}, \quad i \in \mathcal{I} \backslash\{I\}, t \in \mathcal{T} \text {. }
$$

For each $b \in \mathcal{B}$, the block bids are $\left(x_{i b}, p_{i}\right), i \in \mathcal{I}$ and the relation between volumes bid and

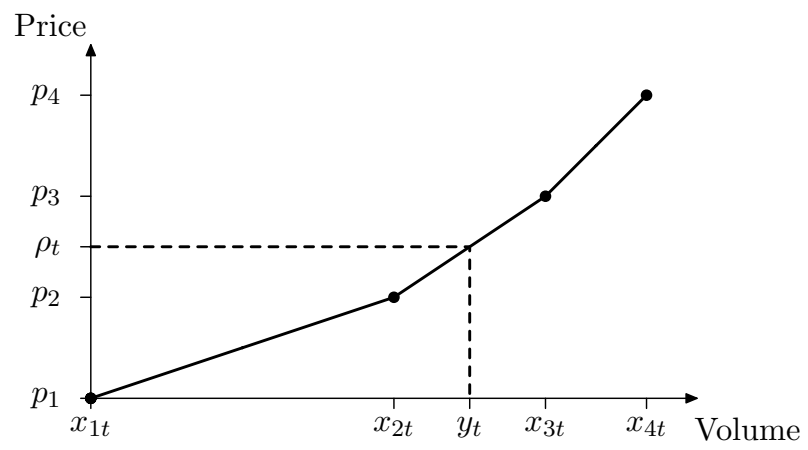

Figure 2.1: Bidding curve of time interval $t \in \mathcal{T}$.

volumes dispatched is

$$
y_{b}=\sum_{j: p_{j} \leq \bar{\rho}_{b}} x_{j b}, \quad b \in \mathcal{B}
$$

i.e. in a given block, the volume dispatched is comprised of the volumes of accepted bids, these being the ones with a price less than the average market price of the block. For example, consider the block $b_{140}=\{1, \ldots, 7\}$. If the volumes bid are $x_{1,140}=(100,100)$ and $x_{2,140}=(50,200)$ and the average market price is $\bar{\rho}_{140}=150$, then the bid $x_{1,140}$ is accepted and the volume dispatched is $y_{140}=100$.

\footnotetext{
${ }^{3}$ Nord Pool does not require bidding curves to be either increasing or decreasing and therefore such constraints may in principle be omitted. Omitting the constraints, however, does not have an influence on the optimal objective function values.
} 


\section{Short-term hydropower production}

Modeling the hydropower production side is relatively standard and follows the lines of for example [24]. This section presents a simple but illustrative model of a small hydropower plant. However, it should be clear that a different modeling of a hydropower plant, for instance a more detailed modeling or a model of a larger plant, is straightforward to combine with the modeling of the bidding process of the preceding section. The plant consists of two reservoirs in a cascade, the upper reservoir being a larger reservoir and the lower reservoir being a smaller one. There is a time delay between the two reservoirs. The combination of time delay and size differences restricts the flexibility of the system. This feature should contribute to understanding the importance of including uncertainty and will be discussed later. To each reservoir corresponds a power station that for ease of exposition has only one generator. hydropower production works as follows. Upstream water reaching the plant flows to the upper reservoir where it is stored until released for generation. When being released, the water from the upper reservoir flows to the lower reservoir and is likewise stored until used for generation. Electricity is generated by changing the potential energy of the water into electrical energy. Water that is not discharged on purpose and used for generation is considered spill. Leaving the plant, the water proceeds downstream. For an illustration, see Fig. 3.1.

To model the production side, let the indices $\mathcal{J}=\{1,2\}$ signify the reservoirs, let the variables

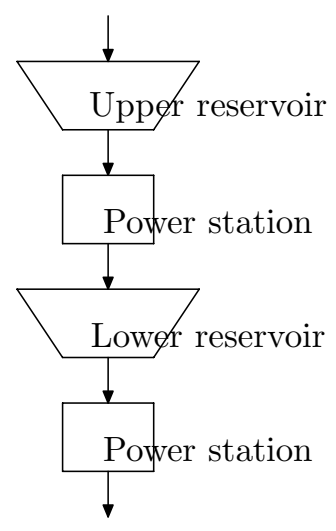

Figure 3.1: Two reservoirs in a cascade.

$u_{j t} \in\{0,1\}, j \in \mathcal{J}, t \in \mathcal{T}$ represent the on/off states of the generators, $w_{j t} \in \mathbb{R}_{+}, j \in \mathcal{J}, t \in \mathcal{T}$ the generation levels and $v_{j t} \in \mathbb{R}_{+}, j \in \mathcal{J}, t \in \mathcal{T}$ the corresponding discharges from the reservoirs. Moreover, let the variables $l_{j t} \in \mathbb{R}_{+}, j \in \mathcal{J}, t \in \mathcal{T}$ be the reservoir storage levels and $r_{j t} \in \mathbb{R}_{+}, j \in \mathcal{J}, t \in \mathcal{T}$ the spill.

Direct costs of hydropower production include only start-up costs as operating costs are negligible. Start-up costs amount to

$$
\sum_{t \in \mathcal{T}} \sum_{j \in \mathcal{J}} S_{j}\left(u_{j t-1}, u_{j t}\right)
$$

with the costs being

$$
S_{j}\left(u_{j t-1}, u_{j t}\right)=c_{j} \max \left\{u_{j t}-u_{j t-1}, 0\right\}, \quad j \in \mathcal{J}, t \in \mathcal{T}
$$

where $c_{j}, j \in \mathcal{J}$ are the start-up costs. Note that the expression is consistent with a mixedinteger linear formulation. Moreover, initial conditions $u_{j 0}=u_{j \mathrm{init}}, j \in \mathcal{J}$ are required. Costs 
also take in opportunity costs of releasing water as the water could be stored and saved for later disposal. Thus, such costs are measured as the value of stored water, this value being available from more long-term models. Here, we have used as water values an average of prices of futures and prices of forward contracts. The opportunity costs are

$$
\sum_{j \in \mathcal{J}}\left(V_{j}\left(l_{j 0}\right)-V_{j}\left(l_{j T}\right)\right)
$$

with

$$
V_{j}\left(l_{j t}\right)=\min _{h \in \mathcal{H}}\left\{d_{h j}^{1} l_{j t}+d_{h j}^{2}\right\}, \quad j \in \mathcal{J}, t \in \mathcal{T}
$$

where $d_{h j}^{1}, d_{h j}^{2}, h \in \mathcal{H}, j \in \mathcal{J}$ are the coefficients of the concave water value functions. Again, the formulation corresponds to a mixed-integer linear formulation.

The following bounds are imposed on the water discharges. The upper reservoir is either not in operation or operated at maximum capacity leading to the constraints

$$
w_{1 t}=u_{1 t} w_{1}^{\max }, \quad t \in \mathcal{T}
$$

where $w_{1}^{\max }$ is the maximum generation level. The lower reservoir, however, can be operated anywhere between minimum and maximum capacity.

$$
u_{2 t} w_{2}^{\min } \leq w_{2 t} \leq u_{2 t} w_{2}^{\max }, \quad t \in \mathcal{T}
$$

where $w_{2}^{\min }$ and $w_{2}^{\max }$ are minimum and maximum generation levels. Similar bounds apply to the discharges, i.e.

$$
v_{j}^{\text {min }} \leq v_{j t} \leq v_{j}^{\max }, \quad j \in \mathcal{J}, t \in \mathcal{T}
$$

Here, $v_{j}^{\text {min }}, j \in \mathcal{J}$ and $v_{j}^{\text {max }}, j \in \mathcal{J}$ are the minimum and maximum discharges. Finally, the storage levels have to adhere to the bounds

$$
l_{j}^{\text {min }} \leq l_{j t} \leq l_{j}^{\text {max }}, \quad j \in \mathcal{J}, t \in \mathcal{T}
$$

where $l_{j}^{\text {min }}, j \in \mathcal{J}$ and $l_{j}^{\text {max }}, j \in \mathcal{J}$ denote minimal and maximal storage levels.

As the reservoir has to balance, reservoir inflow and storage from previous periods either appear as discharge, storage or spill. In case of the upper reservoir the balance equations are

$$
l_{1 t}-l_{1 t-1}+v_{1 t}+r_{1 t}=\nu_{1 t}, \quad t \in \mathcal{T}
$$

where $\nu_{1 t}, t \in \mathcal{T}$ are the inflows from upstream. The initial storage level is $l_{10}=l_{1 \text { init }}$. In case of the lower reservoir, the balance equations are

$$
l_{2 t}-l_{2 t-1}+v_{2 t}+r_{2 t}=v_{1 t-\tau}, \quad t \in \mathcal{T}
$$

where $\tau$ is the time delay between the upper and lower reservoirs. Again, the initial storage level is $l_{20}=l_{2 \text { init }}$.

Ignoring that generation efficiency is not really constant and omitting effects of the reservoir water levels on both the discharge and generation, the two are essentially proportional. This leads to the constraints

$$
w_{j t}=\gamma_{j} v_{j t}, \quad j \in \mathcal{J}, t \in \mathcal{T}
$$


Here, $\gamma_{j}$ are the generation efficiency coefficients.

Imbalances between volumes produced and volumes dispatched in the day-ahead market are settled in a real-time balancing market. Since only the day-ahead market and not the real-time market should act as a de facto spot market, market rules dictate that on average day-ahead exchange should not give rise imbalances. For a hydropower producer this means saving water and planning to release it for generation and bidding in the balancing market is not permitted. In other words, the main problem of short-term planning of a hydropower plant is how much to produce for an operation day and according to the market rules, the solution should be found taking into consideration the day-ahead market primarily. As indeed day-ahead exchange should be in focus, we approximate the balancing effects of the real-time market. We impose a penalty or a reward on imbalances, the penalty being higher than the day-ahead market price and paid if volumes dispatched exceed the volumes produced, i.e. in hours of up-regulation, and the reward being lower than the day-ahead market price and paid in case of the opposite, i.e. in hours of down-regulation. Imposing a penalty or a reward, the producer may still retain flexibility to ramp up or down and bid this flexible capacity into the balancing market close to real-time. Hence, the way of modelling does not reduce the producers' ability to participate in the balancing market. Rather the reverse, penalizing can be seen as an attempt to force the day-ahead market to reflect the physical conditions of the system. Again, making an attempt to avoid deviations in the form of planned imbalances is consistent with how the market operator wants the bidding practice to be. As penalty and reward, we take the average real-time market price of hours where the market has been up-regulated and down-regulated, respectively. Such penalties and rewards would apply if the producer is always regulated in the same direction as the market. This makes sense in that, in reality, a producer is very rarely regulated in the opposite direction of the local market. For a different modelling approach, see also [9]. Let the variables $z_{t}^{+}, z_{t}^{-} \in \mathbb{R}_{+}, t \in \mathcal{T}$ represent the imbalances and let $\mu_{t}^{+}, \mu_{t}^{-}, t \in \mathcal{T}$ denote the corresponding penalty and reward. Then total penalty and reward amount to

$$
\sum_{t \in \mathcal{T}}\left(\mu_{t}^{+} z_{t}^{+}-\mu_{t}^{-} z_{t}^{-}\right)
$$

and the balance constraints are

$$
y_{t}+\sum_{b \in \mathcal{B}: t \in b} y_{b}-\sum_{j \in \mathcal{J}} w_{j t}=z_{t}^{+}-z_{t}^{-}, t \in \mathcal{T}
$$

\section{Day-ahead bidding under uncertainty}

The model of the preceding sections do not take into considerations the uncertainty of the data. In this context, data uncertainty can arise with respect to water inflow and market prices. For obvious reasons, inflow is responsive to the weather situation, however, although this makes inflow forecasting rather complicated during summer, this is not the case in winter. For that reason and since the aim is to analyze uncertainties that relate directly to bidding, we ignore the possibility of inflows to be stochastic. It should be remarked that prices are affected by market conditions that are hard to predict. For a small market participant, the market clearing process is governed by the behavior of other market participants. The information on other market participants is not available and, more often than not, cannot be anticipated. Thus, as market 
prices are determined by clearing the market, prices are unknown at the time of acting in the market. In other words, day-ahead bidding is subject to uncertainty of the market clearing price. Uncertainty can be handled by means of stochastic programming. For an introduction to the subject, see [2], [16] and [25]. A stochastic program is characterized by the partition of decisions into stages according to the flow of information, such that decisions of one stage do not depend on information of subsequent stages. The most obvious objective is based on expectation. Here, decisions are grouped into two, i.e. are to be made before and after observing uncertainty, respectively, and hence, a two-stage stochastic programming model is appropriate. The first stage involves the bidding process, whereas the second stage includes the production aspects. As bids must be submitted to the market before operation, at the time of first-stage decision making, prices are unknown. In contrast, second-stage decisions are deferred to the time of operation and take advantage of the additional information from observing prices. The aim is to obtain the optimal bidding strategies in terms of expected sales and production profit.

To incorporate uncertainty, assume market prices $\left\{\rho_{t}\right\}_{t \in \mathcal{T}}$ form a stochastic process on some probability space. Assume the multivariate distribution is known and in particular that it is discrete with a finite number of realizations $\mathcal{S}=\{1, \ldots, S\}$ referred to as scenarios. Denote the scenario probabilities by $\pi^{s}, s \in \mathcal{S}$ and corresponding market prices by $\left\{\rho_{t}^{s}\right\}_{t \in \mathcal{T}} s \in \mathcal{S}$. Whereas first-stage decisions $x_{i t}, x_{i b}, i \in \mathcal{I}, t \in \mathcal{T}, b \in \mathcal{B}$, i.e. volumes bid, should be independent of future prices, second-stage decisions $y_{t}^{s}, y_{b}^{s}, z_{t}^{+, s}, z_{t}^{-, s}, v_{j t}^{s}, w_{j t}^{s}, l_{j t}^{s} \in \mathbb{R}_{+}, u_{j t}^{s} \in\{0,1\}$, i.e. volumes dispatched as well as production variables, are allowed to depend on the realization of the stochastic prices and are indexed by the scenario superscript $s$ for $s \in \mathcal{S}$. The stochastic program then consists in maximizing the expected profit subject to the constraints. Note that the constraints (2.1)-(2.3) couples first-stage and second-stage decisions through the relation between volumes bid and volumes dispatched whereas the constraints (3.1)-(3.8) applies to second-stage decisions only and model hydropower production. The two-stage stochastic mixed-integer program is then

$$
\begin{aligned}
\max & \sum_{s \in \mathcal{S}} \pi^{s}\left(\sum_{t \in \mathcal{T}} \rho_{t}^{s} y_{t}^{s}+\sum_{b \in \mathcal{B}} \bar{\rho}_{b}^{s} y_{b}^{s}-\sum_{t \in \mathcal{T}}\left(\mu_{t}^{+} z_{t}^{+, s}-\mu_{t}^{-} z_{t}^{-, s}\right)-\right. \\
& \left.\sum_{j \in \mathcal{J}}\left(V_{j}\left(l_{j 0}^{s}\right)-V_{j}\left(l_{j T}^{s}\right)\right)-\sum_{t \in \mathcal{T}} \sum_{j \in \mathcal{J}} S_{j}\left(u_{j t-1}^{s}, u_{j t}^{s}\right)\right) \\
\text { s.t. } & (2.1)-(2.3),(3.1)-(3.8) \\
& x_{i t}, x_{i b} \in \mathbb{R}_{+}, i \in \mathcal{I}, t \in \mathcal{T}, b \in \mathcal{B} \\
& y_{t}^{s}, y_{b}^{s}, z_{t}^{+, s}, z_{t}^{-, s}, v_{j t}^{s}, w_{j t}^{s}, l_{j t}^{s} \in \mathbb{R}_{+}, u_{j t}^{s} \in\{0,1\}, j \in \mathcal{J}, t \in \mathcal{T}, b \in \mathcal{B}, s \in \mathcal{S}
\end{aligned}
$$

It should be remarked that (2.1) and (2.3) can be simplified. Consider a fixed $t \in \mathcal{T}$ and $s \in \mathcal{S}$. Having fixed the price points in advance, the realized market price $\rho_{t}^{s}$ lies between two adjacent points. The remaining price points are irrelevant for determining the volume to be dispatched. Letting $i(t, s)=\max \left\{i \in \mathcal{I}: p_{i} \leq \rho_{t}^{s}\right\}$, the point of dispatch $\left(y_{t}^{s}, \rho_{t}^{s}\right)$ lies on the line segment between $\left(x_{i(t, s) t}, p_{i(t, s)}\right)$ and $\left(x_{i(t, s)+1 t}, p_{i(t, s)+1}\right)$. From this, $(2.1)$ is equivalent to

$$
y_{t}^{s}=\frac{\rho_{t}^{s}-p_{i(t, s)}}{p_{i(t, s)+1}-p_{i(t, s)}} x_{i(t, s)+1 t}+\frac{p_{i(t, s)+1}-\rho_{t}^{s}}{p_{i(t, s)+1}-p_{i(t, s)}} x_{i(t, s) t}, \quad t \in \mathcal{T}, s \in \mathcal{S}
$$


Similarly, letting $i(b, s)=\max \left\{i \in \mathcal{I}: p_{i} \leq \bar{\rho}_{b}^{s}\right\}$, (2.3) can be rewritten as

$$
y_{b}^{s}=\sum_{j \leq i(b, s)} x_{j b}, \quad b \in \mathcal{B}, s \in \mathcal{S}
$$

We operate with two different ways of fixing the prices $p_{i}, i \in \mathcal{I}$, one way being to fix equidistant price points; the other being to fix price points such that the number of realizations day-ahead prices $\rho_{t}^{s}, t \in \mathcal{T}, s \in \mathcal{S}$ between two points is always the same. It should be clear that here the price graduation is more crude in areas where prices are less likely.

To examine the effect on the optimal bidding curves of using a different number of fixed price points, we solved the bidding problem fixing prices in the way that reflects day-ahead price variations, varying this number and compared the resulting curves. In general, the more crude the price graduation, the more crude the bidding curves, although small changes in the number of price points does not alter the bidding curve. Moreover, fixing equidistant price points may induce a rather fine bidding curve as opposed to what is expected when comparing to the one reflecting day-ahead price variations. However, within the interval of realizations of day-ahead prices, the bidding curve derived from equidistant price points is in fact cruder. Still, within the relevant region the optimal curves do not differ much.

\section{Scenario generation}

To describe the behavior of the market clearing electricity prices, the ARIMA methodology is applied, modeling an advanced stochastic process calibrated from historical price profiles. The ARIMA method has been found to be both mathematical sound and accurate in predicting day-ahead electricity prices, an example of this being [6]. Basically, ARIMA processes are a specific class of stochastic processes adopted for analyzing time series and date back to Box and Jenkins [3]. Here, the ARIMA framework will be used to establish a model from which the price scenarios can be generated. A similar approach is seen in [13] considering electricity load scenarios. Other related approaches to modeling electricity prices embrace dynamic regression where load has been included as an explanatory variable, cf. [21], and GARCH models where, as opposed to the ARIMA framework, errors are allowed to be serially correlated and have a non-constant variance, see for example [12]. Among the latest work, long memory processes within the ARFIMA framework has shown to be very promising, cf. [15].

An ARIMA model can be formulated as

$$
\Phi(B) \rho_{t}=\Theta(B) \epsilon_{t}
$$

where $\Phi(B)$ and $\Theta(B)$ are polynomials of the form $\Phi(B)=1-\sum_{i} \phi_{i} B^{i}$ and $\Theta(B)=1-\sum_{i} \theta_{i} B^{i}$ and $B$ denotes the back-shift operator, meaning $B^{i} \rho_{t}=\rho_{t-i}$. Moreover, $\left\{\epsilon_{t}\right\}_{t \in \mathcal{T}}$ is a white noise process, i.e. $\epsilon_{t}, t \in \mathcal{T}$ are independent normally distributed random variables with zero mean and constant variance. The development of the proposed ARIMA model follows the scheme:

1. Identify a statistical model of the observed data.

2. Estimate the parameters of the model.

3. Validate the model. 
4. Use simulation to generate scenarios.

1. For identification an hourly price profile of one year is given. The analysis begins with a careful inspection of the main characteristics of the time series. High frequency, non-constant mean and variance as well as calendar effects and seasonal trends corresponding to daily and weekly periodicity is observed. Creating a trial model, first, the data has to be made stationary making a transformation of the original data necessary. The logarithm is applied to attain a stable variance and the inclusion of the factors $(1-B),\left(1-B^{24}\right)$ and $\left(1-B^{168}\right)$ is used to stabilize the mean. The structure of the polynomials is determined by investigating the autocorrelation and partial autocorrelation functions. In successive trials, refinements can be made based on the residuals of prior models. The ARIMA model is identified as

$$
\begin{aligned}
& (1-B)\left(1-B^{24}\right)\left(1-B^{168}\right) \log \rho_{t}= \\
& \left(1-\theta_{1} B^{1}-\cdots-\theta_{7} B^{7}\right)\left(1-\theta_{23} B^{23}-\theta_{24} B^{24}-\theta_{25} B^{25}-\theta_{47} B^{47}-\theta_{48} B^{48}-\theta_{49} B^{49}\right) \\
& \left(1-\theta_{168} B^{168}\right) \epsilon_{t}
\end{aligned}
$$

2. Having identified a model, the estimators of the parameters can be computed using maximum likelihood optimization. The parameter estimates are given in Table 1.

3. The model is validated checking the assumptions of a white noise process made on the residuals. This is done by studying the autocorrelation and partial autocorrelation functions of the residuals as well as the Ljung-Box statistics. It appears that the model forecasts prices reasonably well, see also Fig. 5.1.

4. Simulated price scenarios $\left\{\rho_{t}^{s}\right\}_{t=1}^{T}, s=1, \ldots, S$ can be generated by extracting the starting values $\rho_{t}, t=-192, \ldots, 0$ and $\epsilon_{t}, t=-223, \ldots, T$ from the historical data and sampling from the i.i.d. normally distributed random variables $\epsilon_{t}, t=1, \ldots, T$. For this purpose, Monte Carlo Simulation has been used, simulating a large number of scenarios. Some descriptive statistics can be found in Table 2. For computational accessibility, however, the number of scenarios has been reduced afterwards using the scenario reduction approach of [13]. An example of the simulation scenarios is shown in Fig. 5.2.

Steps 1-4 are all carried out by the statistical software package SAS, version 8.2.

Although in practice the distribution of the electricity prices is continuous, for computational reasons it is approximated by a discrete distribution. The quality of the approximation is directly linked to the quality of the scenarios, which makes it relevant to evaluate the scenario generation method. With respect to practical performance in-sample and out-of-sample stability as well as stability of the solution can be tested, cf. [17] and [18]. Where the true distribution was needed, back-testing has been done, using historical data instead. The stability analysis gives an indication on how many scenarios to include in order to get a reasonable representation of uncertainty. The stochastic program is found to fulfill the stability requirements in a satisfying way for as few as 10 scenarios.

For illustration purposes a number of demonstration scenarios has also been generated. In all scenarios day-ahead prices are very volatile, i.e. alternating heavily between high and low. An example of the demonstration scenarios is plotted in Fig. 5.3. 
Table 1: Parameter estimates of ARIMA model of hourly day-ahead market prices

\begin{tabular}{llllllll}
\hline Parameter & $\theta_{1}$ & $\theta_{2}$ & $\theta_{3}$ & $\theta_{4}$ & $\theta_{5}$ & $\theta_{6}$ & $\theta_{7}$ \\
\hline Estimates & 0.18576 & 0.14093 & 0.03919 & 0.12922 & 0.09123 & 0.06697 & 0.08096 \\
\hline Parameter & $\theta_{23}$ & $\theta_{24}$ & $\theta_{25}$ & $\theta_{47}$ & $\theta_{48}$ & $\theta_{49}$ & $\theta_{168}$ \\
\hline Estimates & -0.09206 & 0.73214 & -0.09873 & 0.05259 & 0.15267 & 0.05001 & 0.98433 \\
\hline
\end{tabular}

Table 2: Descriptive statistics of hourly day-ahead market prices (in NOK/MWh)

\begin{tabular}{lrrrr}
\hline Sample size & Mean & Std. dev. & Minimum & Maximum \\
\hline 1000 & 174.59 & 11.66 & 141.48 & 221.63 \\
\hline
\end{tabular}

\section{Case study}

This section compares the stochastic formulation of the bidding problem with a corresponding deterministic one. As already stated, the aim is to investigate how including uncertainty into optimization affects the objective value, i.e. the sales and production profit, as well as the solution, i.e. the structure of the bids.

The case study concerns two reservoirs of a small Norwegian hydropower plant located near Trondheim and run by the company TrønderEnergi. The dimensions of the reservoirs and the power stations as well as the operating limits are realistic, although scaled down by a factor ten for computational reasons. Inflows and initial reservoir levels are real data from a typical day, measured in 2005. To simulate day-ahead market prices in 2005, Elspot at Nord Pool has provided real data from 2004 that applied to the Norwegian prize zone, NO2, which is the Trondheim area.

The stochastic programming problem (4.1) is a large-scale mixed-integer linear program containing in the first stage 18300 continuous variables and 1440 constraints and in the second stage for each scenario 590 continuous variables, 48 binary variables and 740 constraints. Different instances were modeled and solved with the linear programming solver from OPL Studio version 3.7 calling CPLEX 9.0 on an Intel Xeon $2.67 \mathrm{GHz}$ processor with 4 GB RAM. The time of compiling and solving the problem varied between around 1 and 3000 seconds since no special effort was made to make the code efficient. In that the problem is a stochastic mixed-integer linear program, special purpose algorithms such as progressive hedging [26] and dual decomposition [4] might prove useful.

To explore the form of the bids, we solved the bidding problem as a stochastic problem with 10 demonstration scenarios and as a deterministic problem, this problem being the corresponding expected value problem formed by replacing random prices by expected ones. Moreover, we computed the expected result of using the expected value solution.

Consider the deterministic bidding problems for one reservoir and for two reservoirs in a cascade. The structure of the solution is simple. In both cases hourly bids are sufficient. Indeed, if the day-ahead price is known in advance, only two hourly bids are relevant. The relevant bids are the ones that determine the part of the bidding curve passing through the point given by the day-ahead price and the optimal dispatch. However, the resulting bidding curve may be very sensitive to changes in the day-ahead price. In extreme scenarios, deviations from the expected 

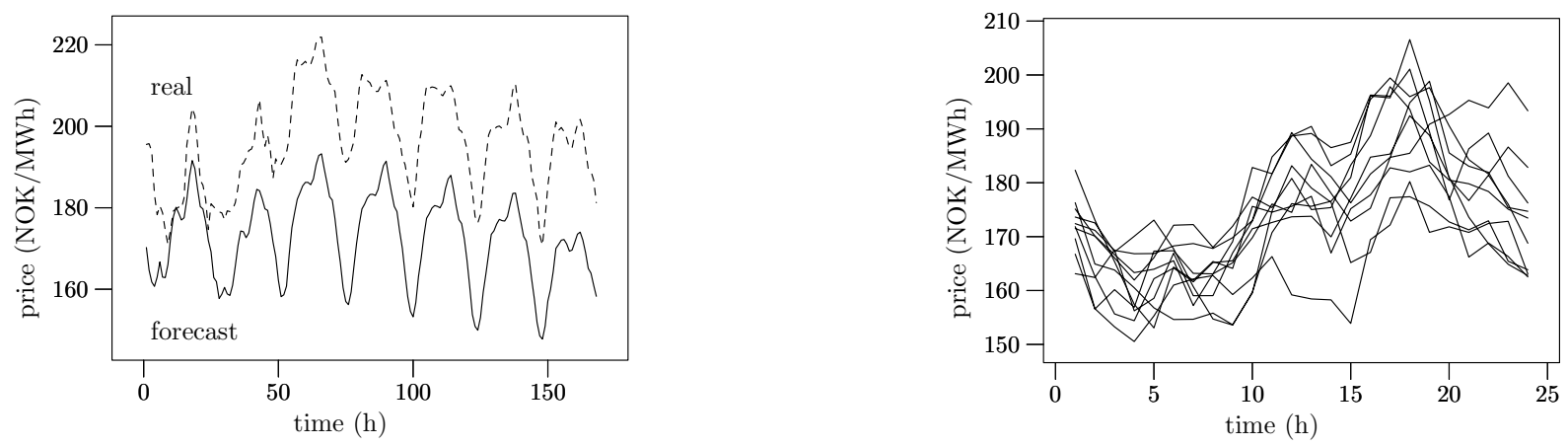

Figure 5.1: Realized vs. forecasted prices.

Figure 5.2: 10 simulation scenarios.

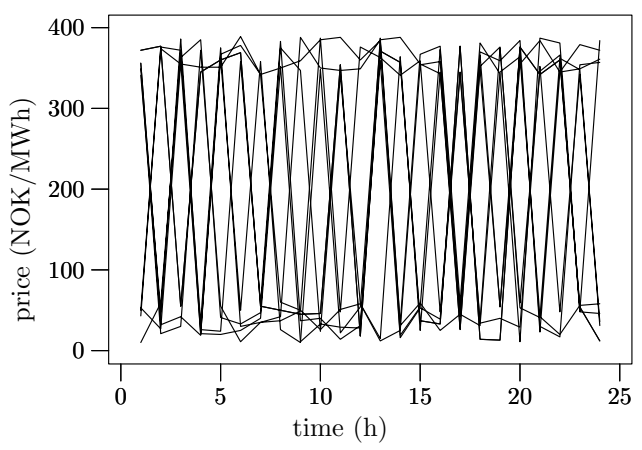

Figure 5.3: 10 demonstration scenarios.

day-ahead price can lead to dispatches far from optimal which means heavy balancing is necessary or in case this is not allowed, dispatches simply cannot be implemented. An example is shown in Table 3, in which the differences (Imbal.) between the total volumes dispatched of hourly bids (H. disp.) and block bids (B. disp.) and the volumes produced (Prod.) are rather large in most scenarios for the given hour shown.

For the stochastic bidding problems, the structure of the solution is more complex. In the one reservoir case, the structure of the solution depends on whether start-up costs are included or not. With no start-up costs, hourly bids are sufficient, whereas with start-up costs both hourly bids and block bids are needed. Hourly bids can follow prices closely. These are, however, accepted independently of each other and do not take into account intertemporalities due to start-ups. Block bids, on the other hand, are accepted as a whole for a number of consecutive hours and thus tend to support a regular production plan with less start-ups. As for one reservoir, in the two reservoir case the structure of the solution depends on whether start-up costs are included or not. The presence of a bottleneck in the system also has an impact on the structure of the solution. A bottleneck occurs when the capacity of the upper reservoir exceeds the capacity of the lower reservoir. Note that water is released from both the upper and the lower reservoir in hours with a high day-ahead price. Due to the bottleneck, too much water released from the upper reservoir may lead to forced releases in the lower reservoir in hours with a low day-ahead price. With no bottleneck, hourly bids are sufficient, whereas, with a bottleneck, both hourly bids and block bids are necessary. A possible explanation is that, as hourly bids are accepted independently, they ignore the intertemporalities generated by dependencies between the reservoirs. On the other hand, since block bids are accepted as a whole for a number of hours, such 
bids can be used as protection against major price fluctuations over time. The time delay between the reservoirs in a cascade shifts the bottleneck problem in time.

To sum up, hourly bids will suffice if the bidding problem is handled as a deterministic problem, whereas block bids are relevant in the corresponding stochastic problem. The difference in the structure of the bids for the expected value problem (EVP) and the stochastic programming problem (SPP) is illustrated in Table 3, where H. disp. refers to the total volume dispatched as hourly bids and B. disp. refers to the total volume dispatched as block bids for the given hour shown. It should be remarked that, by inspecting the objective function value of the problem, if allowing only hourly bids and no block bids as opposed to allowing both kinds of bids, the change is moderate. Nevertheless, as block bids are used more and more frequently in practice, there is a strong motivation for further investigation of including uncertainty into the problem. As regards both the demonstration and simulation scenarios, bidding curves has been drawn in

Table 3: Scenario solutions for a given hour, demonstration scenarios.

The columns H. disp. and B. disp. shows dispatch generated by hourly bids and block bids, separately. Prod. denotes production. The column Imbal. shows imbalances between dispatch and production

\begin{tabular}{rccrrcccr}
\hline \multicolumn{3}{c}{ EEV } & \multicolumn{7}{c}{ SPP } \\
\hline Sce. & H. disp. & B. disp. & Prod. & Imbal. & H. disp. & B. disp. & Prod. & Imbal. \\
\hline 1 & 6.00 & 0.00 & 2.24 & -3.76 & 0.00 & 2.24 & 2.24 & 0.00 \\
2 & 6.00 & 0.00 & 2.24 & -3.76 & 0.00 & 2.24 & 2.24 & 0.00 \\
3 & 0.00 & 0.00 & 1.37 & 1.37 & 0.00 & 2.24 & 2.24 & 0.00 \\
4 & 6.00 & 0.00 & 2.24 & -3.76 & 0.00 & 2.24 & 2.24 & 0.00 \\
5 & 6.00 & 0.00 & 2.24 & -3.76 & 0.00 & 2.24 & 2.24 & 0.00 \\
6 & 0.00 & 0.00 & 1.37 & 1.37 & 0.00 & 2.24 & 2.24 & 0.00 \\
7 & 6.00 & 0.00 & 2.24 & -3.76 & 0.00 & 2.24 & 2.24 & 0.00 \\
8 & 6.00 & 0.00 & 2.24 & -3.76 & 0.00 & 2.24 & 2.24 & 0.00 \\
9 & 6.00 & 0.00 & 2.24 & -3.76 & 0.00 & 2.24 & 2.24 & 0.00 \\
10 & 0.00 & 0.00 & 1.37 & 1.37 & 0.00 & 2.24 & 2.24 & 0.00 \\
\hline
\end{tabular}

Figs. 6.1 and 6.2, respectively. The curves are shown for the both the deterministic problem (det.), cf. the dashed lines, and for the stochastic problem (sto.) with 10 scenarios, cf. full-drawn lines. The vertical lines represent block bids, whereas the other ones correspond to hourly bids. Although the bidding curves appear as piece-wise constant ones, the curves are in fact piecewise linear. Being close to piece-wise constant is consistent with the current bidding practice of the hydropower producer of this application and means that, between certain price levels the producer behaves as price insensitive. The figures also illustrate the discussion above. Consider Fig. 6.1. For an expected day-ahead price of $261.90 \mathrm{NOK} / \mathrm{MWh}$, the dispatch is the same for the deterministic and the stochastic case, i.e. 2.24 MWh. However, as already stated, the deterministic bidding curves are very sensitive to changes in the day-ahead price. If, for instance, the price turns out to be $28.00 \mathrm{NOK} / \mathrm{MWh}$ (scenario 10) or $389.00 \mathrm{NOK} / \mathrm{MWh}$ (scenario 1 ), the dispatch must be very small $(0.00 \mathrm{MWh})$ or very large $(6.00 \mathrm{MWh})$, respectively, which calls for balancing and increased costs. The same situation is valid in Fig. 6.2.

To analyze how including uncertainty into optimization affects the production and sales profit, 


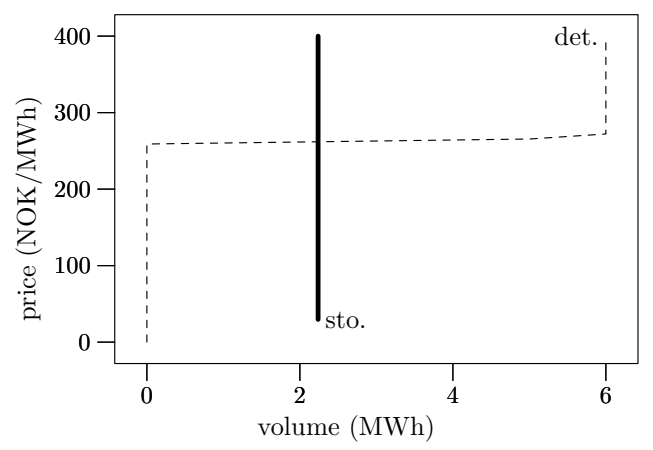

Figure 6.1: Bidding curves for a given hour, demonstration scenarios.

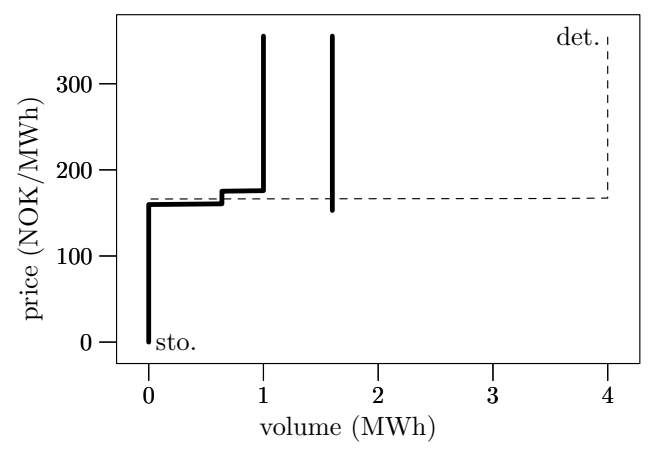

Figure 6.2: Bidding curves for a given hour, simulation scenarios.

we solved the stochastic programming version of the bidding problem with both the demonstration scenarios and the simulation scenarios, in the last case varying the number of scenarios. For the demonstration scenarios, we have used equidistant price points, whereas, for the simulation scenarios, we have used price points that reflect the variation in realized day-ahead prices. We recorded the optimal value of the stochastic program as well as the expected result of using the expected value solution (EEV). To compare the two, we calculated the difference, referred to as the value of using the stochastic solution (VSS). Averages of 10 different runs are reported in Tables 4 and 5. The value of the stochastic solution measures the effect of including stochastic prices explicitly into the bidding problem rather than simply using expected prices. We focus on the computational results of using the simulated scenarios. It follows that the value of the stochastic solution is on average $7.93 \%$. On a per day basis the gains of solving the stochastic program rather than the expected value problem may seem moderate in money terms. Still, recall that the dimension of the power plant has been scaled down by a factor ten compared to realistic data. Hence, solving the problem every day of the year, it seems that significant profits can be earned by applying stochastic programming.

Table 4: Computational results for demonstration scenarios

\begin{tabular}{cccl}
\hline S & Opt. val. & EEV & VSS \\
\hline 10 & 34801.51 & 33821.17 & 980.34 \\
\hline
\end{tabular}

Table 5: Computational results for simulated scenarios

\begin{tabular}{rccc}
\hline \multicolumn{1}{c}{ S } & Opt. val. & EVP & VSS \\
\hline 10 & 33395.45 & 30992.49 & 2402.96 \\
30 & 33420.60 & 30676.07 & 2744.53 \\
50 & 33405.75 & 30805.37 & 2600.39 \\
100 & 33419.60 & 30566.44 & 2853.17 \\
\hline
\end{tabular}




\section{Acknowledgments}

The authors would like to thank Bernhard Kvaal, Gunnar Aronsen and Lars Olav Hoset at TrønderEnergi for indispensable information on theory and practice of both hydropower production and bidding, Matthias P. Nowak at SINTEF for fundamental understandings and suggestions on how to structure the paper, Olav Bjarte Fosso and Michael M. Belsnes at Norwegian University of Science and Technology for valuable discussions of the problem and its relevance, Nina Detlefsen and Frede Aakmann Tøgersen at Forskningscenter Foulum as well as Preben Blæsild and Anders Holst at the University of Aarhus for assistance concerning the time series analysis.

\section{References}

[1] E.J. Anderson, A.B. Philpott, Optimal offer construction in electricity markets, Mathematics of Operations Research 27 (1) (2000) 82-100.

[2] J.R. Birge, F. Louveaux, Introduction to Stochastic Programming, Springer, New York, 1997.

[3] G.E.P. Box, G.M. Jenkins, Time Series Analysis, Holden Day, 1970.

[4] C.C. Caröe, R. Schultz, Dual decomposition in stochastic integer programming, Operations Research Letters 24 (1998) 37-45.

[5] A.J. Conjeno, F.J. Nogales, J.M. Arroyo, Price-taker bidding strategy under price uncertainty, IEEE Transactions on Power Systems 17 (4) (2002) 1081-1088.

[6] J. Contreras, R. Espinola, F.J. Nogales, A.J. Conejo, ARIMA models to predict next-day electricity prices, IEEE Transactions on Power Systems 18 (3) (2003) 1014-1019.

[7] G.B. Dantzig, G. Infanger, Intelligent control and optimization under uncertainty with application to hydro power, European Journal of Operations Research 97 (1997) 396-407.

[8] N. Flatabø, A. Haugstad, B. Mo, O.B. Fosso, Short-term and medium-term generation scheduling in the Norwegian hydro system under a competitive power market structure, VIII SEPOPE'02, Brasil, 2002.

[9] S.-E. Fleten, E. Pettersen, Constructing bidding curves for a price-taking retailer in the Norwegian electricity market, IEEE Transactions on Power Systems 20 (2) (2005) 701-708.

[10] O.B. Fosso, M.M. Belsnes, Short-term Hydro Scheduling in a Liberalized Power System, Proceedings of the International Conference on Power System Technology, 21-24 Nov 2004, Singapore.

[11] O.B. Fosso, A. Gjelsvik, A. Haugstad, B. Mo I. Wagensteen, Generation scheduling in a deregulated system. The Norwegian case, IEEE Transactions on Power Systems 14 (1) (1999) $75-81$.

[12] R.C. Garcia, J. Contreras, M.V. Akkeren, J.B. C. Garcia, A GARCH forecasting model to predict day-ahead electricity prices, IEEE Transactions on Power Systems 20 (2) (2005) $867-874$. 
[13] G. Gröwe-Kuska, K.C. Kiwiel, M.P. Nowak, W. Römisch, I. Wegner, Power management under uncertainty by lagrangian relaxation, proceedings of the 6 th international conference probabilistic methods applied to power systems PMAPS 2000 2, INESC Porto, 2000.

[14] N. Gröwe-Kuska, K.C. Kiwiel, M.P. Nowak, W. Römisch, I. Wegner, Power management in a hydro-thermal system under uncertainty by Lagrangian relaxation, in: C. Greengard, A. Ruszczynski (Eds.), Decision Making under Uncertainty: Energy and Power, IMA Volumes in Mathematics and its Applications Vol. 128, Springer-Verlag, New York, 2002, 39-70.

[15] N. Haldrup, M.O. Nielsen. A regime switching long memory model for electricity prices (2004) (In Press, Journal of Econometrics, available http://dx.doi.org/10.1016/j. jeconom.2005.07.021).

[16] P. Kall, S.W. Wallace, Stochastic Programming, Wiley, Chichester (1994).

[17] M. Kaut, S.W. Wallace, Stability analysis of a portfolio management model based on the conditional value-at-risk measure (2003) (Downloadable from website http://work. michalkaut.net/CV_and_study/CVaR_stability_abstract.htm.

[18] M. Kaut, S.W. Wallace, Evaluation of scenario-generation methods for stochastic programming (2003) (Downloadable from website http://work.michalkaut.net/CV_and_study/ SG_evaluation_abstract.htm).

[19] P. Lino, L.A. Barroso, M.V.F. Pereira, R. Kelman, M.H.C. Fampa, Bid-based dispatch of hydro-thermal systems in competitive markets, Annals of Operations Research 120 (2003) 81-97.

[20] X. Ma, F. Wen, J. Liu, Towards the development of risk-constrained optimal bidding strategies for generation companies in electricity markets, Electric Power Systems Research 73 (2005) 305-312.

[21] F.J. Nogales, A.J. Conejo and R. Espinola, Forecasting next-day electricity prices by time series models, IEEE Transactions on Power Systems 17 (2) (2002) 342-349.

[22] M.P. Nowak, R. Nürnberg, W. Römisch, R. Schultz, M. Westphalen, Stochastic programming for power production and trading under uncertainty, in: W. Jäger, H.-J. Krebs (Eds.), Mathematics - Key Technology for the Future, Springer, Berlin, 2003, pp. 623-636.

[23] P. Oliveira, S. McKee, C. Coles, Optimal scheduling of a hydro thermal power generation system, European Journal of Operations Research 71 (1993) 334-340.

[24] A.B. Philpott, M. Craddock, H. Waterer, Hydro-electric unit commitment subject to uncertain demand, European Journal of Operations Research 125 (2000) 410-424.

[25] A. Prekopa, Stochastic Programming, Kluwer, Dordrecht (1995).

[26] R.T. Rockafellar, L.A. Wets. Scenarios and policy aggregation in optimization under uncertainty, Mathematics of Operations Research 16 (1991) 119-147. 
[27] Z.K. Shawwash, T.K. Siu, S.O. Russel, The B.C. Hydro short term hydro scheduling optimization model, IEEE Transactions on Power Systems 15 (3) (2000) 1125-1131.

[28] S.W. Wallace, S.-E. Fleten, Stochastic programming models in energy, in: A. Ruszczynski, A. Shapiro (Eds.), Stochastic Programming, Handbooks in Operations Research and Management Science 10, Elsevier, North-Holland, 2003, pp. 637-677.

[29] F.S. Wen, A.K. David, Strategic bidding for electricity supply in a day-ahead energy market, Electric Power Systems Research 59 (2001) 197-206. 